\title{
Antioxidant property of leaves and calluses extracts of in-vitro grown 5 different Ocimum species
}

\author{
Hyuk Song • Prem Kumar · Girija Arivazhagan · Sang-Il Lee $\cdot$ Hyung Moon Yoon · Ick Hee Kim · Hyuk Jung Kwon \\ Jong Moon Kim · Faruck Lukmanul Hakkim
}

Received: 7 September 2012 / Accepted: 14 September 2012

(c) Korean Society for Plant Biotechnology

\begin{abstract}
In this study, the antioxidant property of leaf and callus extracts of five selected in vitro grown Ocimum species (Ocimum sanctum, Ocimum kilimandscharicum, Ocimum gratissimum, Ocimum basilicum, and Ocimum americanum) and their respective callus extracts was investigated. The callus cultures were successfully initiated on Murashige and Skoog (MS) medium supplemented with 2,4-dichlorophenoxy acetic acid (2,4-D) $(1 \mathrm{mg} \cdot \mathrm{L})$ combined with different concentrations $(0.1-0.4 \mathrm{mg} \cdot \mathrm{L})$ of kinetin as plant growth regulators. Total phenolic contents were estimated using the Folin-Ciocalteu reagent. 1,1-diphenyl-2-
\end{abstract}

H. Song $\cdot$ F. L. Hakkim

Department of Animal \& Food Bioscience, Konkuk University, Chungju 380-701, Republic of Korea

P. Kumar • G. Arivazhagan

School of Biotechnology and Genetic Engineering, Bharathiar

University, Coimbatore, Tamilnadu, India.

S.-I. Lee

Department of Biotechnology, College of Biomedical and Health

Science, Konkuk University, Chungju 380-701, Republic of

Korea

H. M. Yoon

Department of Orthopaedic Surgery, School of Mdedicine, Konkuk University, Chungju 380-701, Republic of Korea

\section{H. Kim}

Department of Surgery, School of Medicine, Konkuk University, Chungju 380-701, Republic of Korea

\section{H. J. Kwon}

Department of Family medicine, School of Medicine, Konkuk University, Chungju 380-701, Republic of Korea

\section{J. M. Kim}

Department of Rehabilitation, School of Medicine, Konkuk University, Chungju 380-701, Republic of Korea

F. L. Hakkim (ه)

DARIS Centre for Scientific Research and Technology Development,

University of Nizwa, Oman

e-mail: biohakkim@gmail.com picrylhydrazyl (DPPH) radical scavenging, ferric reducing antioxidant power, $\mathrm{Fe}^{2+}$ chelating activity, and $\beta$-carotenelinoleic acid bleaching assays were used to determine the biological effects of the extracts. Interestingly, all the callus extracts exhibited significant $(p<0.05)$ increase in phenolic contents and antioxidant activity. Furthermore, a liner correlation was obtained between the total phenolic contents and free radical scavenging activity $\left(\mathrm{R}^{2}=0.783\right)$. The extracts of leaves and calluses of Ocimum species exhibited activity in all the in vitro antioxidant assays, but its extent was less potent that the positive controls butylated hydroxyl anisole (BHA) and ascorbic acid. A higher accumulation of phenolics in the callus extracts suggests that isolation of high-concentration materials with antioxidant activivity is possible from in vitro callus cultures rather than field-grown plant organs. Furthermore, these extracts may be used as an effective preservative in the food industry.

Keywords DPPH, Ocimum kilimandscharicum, Ocimum gratissimum, Ocimum basilicum, Ocimum americanum, phenolics

\section{Introduction}

Chemical constituents that have have antioxidant properties in high concentrations in plants can play a role in the prevention of various degenerative diseases (Velioglu et al. 1998). In recent years, research efforts have been focused on the possibilities utilizing natural sources of bioactive compounds for the dietary management of certain chronic diseases such as diabetes, obesity, cardiovascular diseases, and cancer (Vadivel and Biesalski 2011). Throughout the past history, spices and herbs have been used to flavor foods and beverages, as well as for medicinal purposes. The preservative effect of many plant species and herbs 
suggests the presence of antioxidative and antimicrobial constituents (Hirasa and Takemasa 1998). Besides fruits and vegetables, herb or medicinal plants are recommended at present as optimal sources of such components. The supplementation of the human diet with herbs, containing especially high amounts of compounds capable of deactivating free radicals, may have beneficial effects (Lutomski 2002). Among the important constituents in plants, phenolic compounds have been reported to be involved in cell defence against free radicals (Szeto et al. 2002). A number of phenolic compounds with strong antioxidant and antimicrobial activities have been identified in plants, especially in those belonging to the Lamiaceae family. They are rising in popularity and in interest among food manufacturers, as consumers increasingly seek after functional foods with specific health effects (Ozkan et al., 2003). Generally, antioxidant activity is assessed by 1,1diphenyl-2-picrylhydrazyl (DPPH), ferric reducing antioxidant power (FRAP), $\mathrm{Fe}^{2+}$ chelating activity assay, and $\beta$-carotenelinoleic acid bleaching methods (Gyamfi et al. 1999; Benzie and Strain 1996; Dinis et al. 1994; Koleva et al. 2002). DPPH method uses a free radical scavenging ability of chemicals or materials using in animal and plant cells, and FRAP method assessed the total reducing power of the electron donating substances from the chemicals or tested materials in the cultured cell. $\mathrm{Fe}^{2+}$ chelating activity can be measured the activity of $\mathrm{Fe}^{2+}$-ferrozine complex formation, indicates oxidation of molecules, and $\beta$-carotenelinoleic acid bleaching method can determine the colour bleaching level of the tested samples on $\beta$-carotene. These methods are widely used to determine the ability of antioxidant.

The genus Ocimum, a member of the Lamiaceae family, contains 200 species of herbs and shrubs (Simon et al., 1999). This species boasts of long history as culinary herbs, due to its foliage adding a distinctive flavour to many foods. It is also a source of aromatic compounds and essential oils containing biologically active constituents that possess insecticidal and nematicidal properties (Chatterjee et al. 1982; Deshpande and Tipins 1977). In our previous study, we selected the ocimum species such as Ocimum sanctum, Ocimum kilimandscharicum, Ocimum gratissimum, Ocimum basilicum, and Ocimum americanum to test their antioxidant ability and found significant free radical scavenging ability (Hakkim et al. 2008). Generally, the callus biomass accumulates high amounts of phenolics compared to their in vivo counter parts. We demonstrated this from a previous study, in which Ocimum sanctum leaf callus cultures showed an accumulation of maximum level of phenolics than in vivo leaves (Hakkim et al. 2007).

Although we showed that leaves of Ocimum kilimandscharicum, Ocimum gratissimum, Ocimum basilicum, and Ocimum americanum have antioxidant (AO) ability, whether their respective callus biomass contains AA remains elusive. In this study, we included the $O$. Sanctum callus cultures also as a positive control. To the best of our knowledge, our study is the first to comparatively analyze the in vivo and in vitro AO ability of different Ocimum species. Since the Ocimum species extract is one of the important herbal formulations in ayurvedic medicine as a tonic for treating many diseases, this study adds further value for the possible use of these plants, especially the in vitro-induced callus extract as a food additive.

\section{Materials and methods}

Plant source

The plant materials of Ocimum sanctum, Ocimum americanum, Ocimum basilicum, Ocimum kilimandsharicum, Ocimum gratissimum were collected from local farms and were grown at the department of Biotechnology, Bharathiar University, and Coimbatore, India. Green houseplants were irrigated to pot daily and maintained at day/night temperatures of $27-33$ and $20-25^{\circ} \mathrm{C}$ respectively. The leaves was harvested from fully matured plants and kept for air-drying at room temperature for 7 days for chemical analysis.

\section{Callus induction}

Leaf explants from 1-year-old Ocimum sanctum, Ocimum americanum, Ocimum basilicum, Ocimum kilimandsharicum, Ocimum gratissimum plants were surface-sterilized for 3-5 min in Tween 80 and for 3 min with $0.1 \%(w / v) ~ H g C l$ and then rinsed three times with sterilized distilled water. Sterilized explants were cut into $1 \mathrm{~cm}$-long pieces and cultured on Murashige and Skoog (MS) (Murashige and Skoog 1962) medium containing $3 \%$ sucrose (analytical grade) as a carbon source and $0.8 \%$ agar (Himedia Laboratories, Mumbai, India) for gelling, and growth regulators such as 2,4-D $(1 \mathrm{mg} \cdot \mathrm{L})$ combined with different concentrations $(0.1-0.4 \mathrm{mg} \cdot \mathrm{L})$ of kinetin were used individually. The cultures were maintained at $25 \pm 2{ }^{\circ} \mathrm{C}$ under $12 \mathrm{hr}$ alternative dark and light (1,000-2,000 lux) cycles. Culture responses of each explant for callus induction were recorded after 8 weeks of culture. 


\section{Extraction (In Vivo)}

The five varieties of Ocimum species plant leaves were collected, shade dried for a period of 10 days, grounded into powder. $10 \mathrm{mg}$ of each powdered material was extracted twice with $500 \mathrm{ml}$ of methanol continuously for $6 \mathrm{~h}$ using the soxhlet apparatus. Thereafter, the resulting methanolic extract was reduced in vacuo $\left(40^{\circ} \mathrm{C} ; \mathrm{N}_{2}\right.$ stream), freeze-dried and stored at $4{ }^{\circ} \mathrm{C}$ until further use in the experiment.

\section{Extraction (In vitro)}

The callus induced on MS medium fortified with 2,4-D (1 $\mathrm{mg} \cdot \mathrm{L}$ ) combined individually with varying concentrations (0.1-0.4 $\mathrm{mg} \cdot \mathrm{L})$ of kinetin were harvested, dried, and the dry weights were determined (Szabo et al. 1999). Extraction was performed as described above.

\section{Determination of total phenolic Content}

Total phenolic contents were determined as Gallic acid equivalents (GAE) (Singleton et al. 1999). The dry extract $(30 \mathrm{mg})$ was diluted in $1 \mathrm{ml}$ of methanol, and $100 \mu \mathrm{l}$ of the solution was transferred to a $10 \mathrm{ml}$ volumetric flask, to which $0.5 \mathrm{ml}$ undiluted Folin-Ciocalteu reagent was added. After a minute, $1.5 \mathrm{ml}$ of $20 \%$ (w/v) $\mathrm{Na}_{2} \mathrm{CO}_{3}$ was added and the volume reconstituted in $10 \mathrm{ml}$ of water. After one hour incubation at $25^{\circ} \mathrm{C}$, the absorbance was measured at $760 \mathrm{~nm}$ and compared to a pre-prepared Gallic acid calibration curve.

\section{DPPH radical scavenging assay}

The ability of the extracts to scavenge the DPPH radical was assessed spectrophotometrically (Gyamfi et al., 1999). Briefly, $50 \mu \mathrm{l}$ aliquot of the extract $(1 \mathrm{mg} \cdot \mathrm{ml})$ was mixed with $450 \mu \mathrm{l}$ Tris-Hcl buffer $(50 \mathrm{mmol} \cdot \mathrm{L}, \mathrm{pH}$ 7.4) and 1 $\mathrm{ml}$ DPPH $(0.1 \mathrm{mmol} \cdot \mathrm{L}$, in methanol), the resultant absorbance was recorded at $517 \mathrm{~nm}$ after 30 min incubation at $37^{\circ} \mathrm{C}$. The percentage of inhibition was calculated from the following equation:

Percentage of inhibition $=\left[\left(\mathrm{A}_{0}-\mathrm{A}_{1}\right) / \mathrm{A}_{0}\right] \mathrm{X} 100$

where $A_{0}$ was the absorbance of the control (blank, without extract), and $A_{1}$ was the absorbance in the presence of the extract. Butylated hydroxy anisole (BHA) $(0.2 \mathrm{mg} \cdot \mathrm{ml})$ and ascorbic acid $(0.2 \mathrm{mg} \cdot \mathrm{ml})$ served as control.
Ferric reducing antioxidant power (FRAP) assay

The FRAP, a method for measuring the total reducing power of the electron donating substances, was assessed according to Benzie and Strain (1996). Briefly, $6 \mathrm{ml}$ of freshly prepared working FRAP reagent $(0.1 \mathrm{M}$ acetate buffer: $0.02 \mathrm{M} \mathrm{FeCl}_{3}: 0.01 \mathrm{M} \mathrm{TPTZ}=10: 1: 1$ ) was mixed with $20 \mu \mathrm{l}$ of extract sample $(1 \mathrm{mg} \cdot \mathrm{ml})$. The absorbance at $593 \mathrm{~nm}$ was recorded after a $30-$ min incubation at $37^{\circ} \mathrm{C}$. (BHA) $(0.2 \mathrm{mg} \cdot \mathrm{ml})$ and ascorbic acid $(0.2 \mathrm{mg} \cdot \mathrm{ml})$ served as control.

\section{$\mathrm{Fe}^{2+}$ chelating activity assay}

The chelating activity of extracts for ferrous ions $\mathrm{Fe}^{2+}$ was determined (Dinis et al., 1994). Briefly, to $0.5 \mathrm{ml}$ of the extract $(1 \mathrm{mg} \cdot \mathrm{ml}), 1.6 \mathrm{ml}$ of distilled water and $0.05 \mathrm{ml}$ of $\mathrm{FeCl}_{2}(2 \mathrm{mM})$ was added and after $30 \mathrm{~s}, 0.1 \mathrm{ml}$ ferrozine $(5 \mathrm{mM})$ was added. Then the reaction mixture was incubated for $10 \mathrm{~min}$ at room temperature, and the absorbance of the $\mathrm{Fe}^{2+}$-Ferrozine complex was measured at $562 \mathrm{~nm}$. A lower absorbance indicates a higher chelating power. The chelating activity of the extracts on $\mathrm{Fe}^{2+}$ was compared with that of EDTA $(0.01 \mathrm{mM})$ and Citric acid $(0.025$ M).

The chelating activity was calculated according to the following equation:

Chelating activity $(\%)=\left[\left(\mathrm{A}_{0}-\mathrm{A}_{1}\right) / \mathrm{A}_{0}\right] \mathrm{X} 100$

where $A_{0}$ was the absorbance of the control (blank, without extract) and $A_{1}$ was the absorbance in the presence of the extract.

\section{ß-carotene-linoleic acid bleaching}

The ability of the extracts to prevent the bleaching of $\beta$ -carotene was assessed (Koleva et al. 2002). Briefly, 0.2 $\mathrm{mg} \beta$-carotene in $1 \mathrm{ml}$ chloroform, $20 \mathrm{mg}$ of linoleic acid and $200 \mathrm{mg}$ of Tween 20 were transferred into a roundbottom flask. After removal of chloroform, $50 \mathrm{ml}$ distilled $\mathrm{H}_{2} \mathrm{O}$ was added, and the resulting mixture was stirred vigorously. $6 \mathrm{ml}$ aliquots of the emulsion were transferred to tubes containing either $50 \mu \mathrm{l}(1 \mathrm{mg} \cdot \mathrm{ml})$ extract or 50 $\mu \mathrm{l}(0.2 \mathrm{mg} \cdot \mathrm{ml})$ BHA or $50 \mu \mathrm{l}(0.2 \mathrm{mg} \cdot \mathrm{ml})$ ascorbic acid. After mixing, an aliquot was transferred into a cuvette, and the absorbance $\left(\mathrm{Abs}^{\circ}\right)$ at $470 \mathrm{~nm}$ was recorded. The remaining samples were placed in a water bath at $50^{\circ} \mathrm{C}$ for a period of $2 \mathrm{~h}$. Thereafter, the absorbance of each sample 
was measured at $470 \mathrm{~nm}\left(\mathrm{Abs}^{120}\right)$. The data $(\mathrm{n}=3)$ are presented as antioxidant activity \% (AA\%) values, calculated using the equation:

$$
\begin{aligned}
\mathbf{A A} \%= & {\left[1-\left(\mathrm{Ab}_{\text {sample }}^{0}-\mathrm{Abs}^{120}{ }_{\text {sample }}\right)\right.} \\
& /\left(\mathrm{Ab}_{\text {control }}^{0}-\mathrm{Abs}^{120}{ }_{\text {control })}\right) \times 100
\end{aligned}
$$

Statistical Analysis

All data were expressed as mean $\pm \mathrm{SD}$ of the number of experiments $(\mathrm{n}=3)$. Two-way analysis of variance (ANOVA) was performed using SPSS software to analyze the statistical significance. The level of significance was considered at $\mathrm{p}<0.05$.

\section{Results and discussion}

Callus Induction

Most naturally-occurring antioxidants are secondary metabolites which include alkaloids, flavonoids, and phenolic acids. The antioxidant activity of these compounds is predominantly determined by their structures, particularly the presence of hydroxyl groups over the aromatic nucleus. While the intact plant organs contain secondary metabolites, the culture of undifferentiated cells (calli) are a potent source for the production of wide variety of secondary metabolites (Verpoorte and Alferman, 2000). In this study, the callus cultures were successfully induced from 5 different Ocimum species of leaves, grown on MS medium containing 2,4-D $(1 \mathrm{mg} \cdot \mathrm{L})$ combined with kinetin $(0.1-0.4 \mathrm{mg} \cdot \mathrm{L})$, to assess its chemical composition and antioxidant ability. Interestingly, the culture response was found to vary according the concentration of kinetin supplementation (Table 1). O. sanctum showed maximum response with supplementation of 2,4-D $(1 \mathrm{mg} \cdot \mathrm{L})$ and kinetin $(0.1 \mathrm{mg} \cdot \mathrm{L})$, while $O$. basilicum showed maximum response with supplementation of 2,4-D $(1 \mathrm{mg} \cdot \mathrm{L})$ and kinetin $(0.2 \mathrm{mg} \cdot \mathrm{L})$. Furthermore, O. americanum, O. gratissimum, and $O$. kilimandscharicum exhibited maximum response with supplementation of 2,4-D $(1 \mathrm{mg} \cdot \mathrm{L})$ and kinetin $(0.3$ $\mathrm{mg} \cdot \mathrm{L})$. This variation in the culture response may be due to the difference in inter-species metabolism upon kinetin treatment.

Total phenolic content

In the present study, we evaluated the total phenolic content of the methanolic extract of the five varieties of Ocimum species in leaves and their respective callus cultures induced from each explants. Phenolic contents ranged from 66.4 to $112.3 \mathrm{mg} \mathrm{GAE} \cdot \mathrm{gm}$ of dry extract in leaf extracts and from 84.6 to $153.4 \mathrm{mg} \mathrm{GAE} \cdot \mathrm{gm}$ of dry extract in leaf callus culture extracts (Fig. 1). This demonstrates that callus cultures have the capacity to retain significant amounts of phenolics rather than their in vivo counter parts. As the callus growing in a nutrient-rich culture medium is undoubtedly exposed to a more carbon influx than the field-grown plant parts, it may influence the metabolic flux for the biosynthesis of elevated levels of phenolics. Similarly, the accumulation of phenolics was reported in Salvia officinalis callus cultures, where the level of phenolics in callus is based on the abundance of carbon sources in the medium (Paula et al. 2002).

\section{1,1-Diphenyl-2-picrylhydrazyl (DPPH) free radical scavenging activity}

Free radicals (DPPH) are well known to cause auto oxidation of unsaturated lipids in foods (Kaur and Perkins 1991). Determination of scavenging stable DPPH was a very fast method to evaluate the antioxidant activity of the extracts. With this method, it was possible to determine

\begin{tabular}{|c|c|c|c|c|c|c|}
\hline \multirow{2}{*}{\multicolumn{2}{|c|}{$\begin{array}{c}\text { Growth regulators } \\
(\mathrm{mg} / \mathrm{l})\end{array}$}} & \multicolumn{5}{|c|}{$\%$ of culture response } \\
\hline & & \multirow[t]{2}{*}{ O. sanctum } & \multirow[t]{2}{*}{ O. basilicum } & \multirow[t]{2}{*}{ O. americanum } & \multirow[t]{2}{*}{ O.gratissimum } & \multirow[t]{2}{*}{ O. kilimandcharicum } \\
\hline $2,4 \mathrm{D}$ & + kinetin & & & & & \\
\hline 1.0 & 0.1 & $99.4 \pm 2.3^{*}$ & $70.1 \pm 2.1$ & $80.5 \pm 2.1$ & $73.5 \pm 2.4$ & $70.1 \pm 2.7$ \\
\hline 1.0 & 0.2 & $75 \pm 2.4$ & $99.5 \pm 3.4^{*}$ & $75 \pm 2.3$ & $70 \pm 2.2$ & $78 \pm 3.2$ \\
\hline 1.0 & 0.3 & $98.5 \pm 2.6$ & $70 \pm 3.2$ & $99.1 \pm 2.6^{*}$ & $96 \pm 3.1^{*}$ & $99.4 \pm 3.4^{*}$ \\
\hline 1.0 & 0.4 & $50 \pm 2.8$ & $55 \pm 2.4$ & $58 \pm 3.1$ & $50 \pm 2.6$ & $53 \pm 2.6$ \\
\hline
\end{tabular}

Table 1 Effect of various concentrations of auxins on callus induction using leaves of 5 different Ocimum species

Data were collected after 8 weeks of culture. Values are given as mean \pm SD for each three triplicates. $*$ indicates the level of significance at $\mathrm{p}<0.05$ compare to other auxin combination supplementation. 


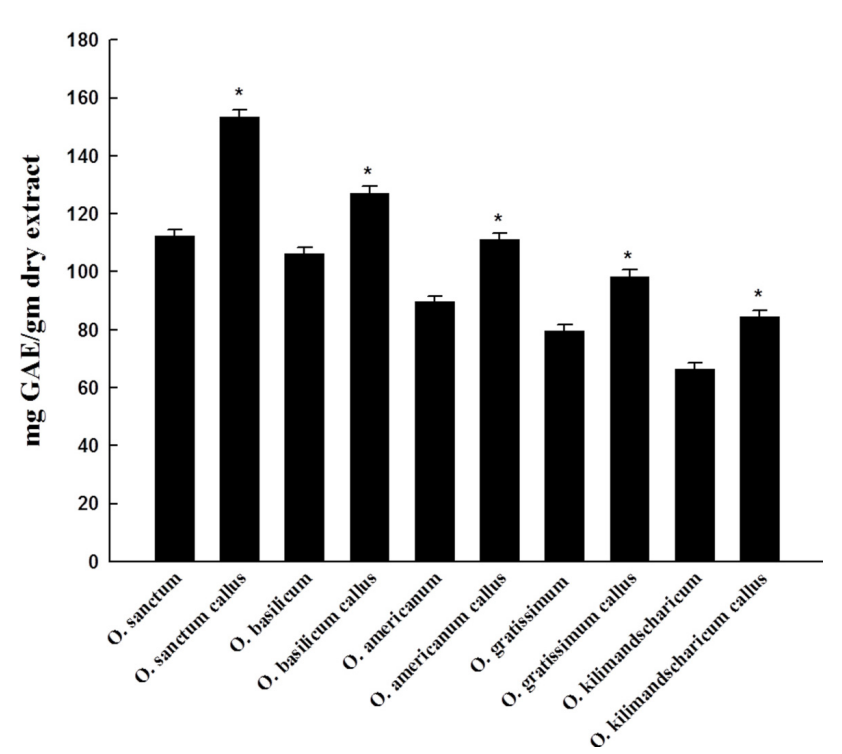

Fig. 1 Total phenolic contents of five different Ocimum species leaves and their in vitro leaf callus extracts. Data are presented as mean $\pm \mathrm{SD}$ of each of three replicates $(n=3)$. * indicates a significant $(\mathrm{p}<0.05)$ increase in callus extract compare to their respective in vivo leaf extracts

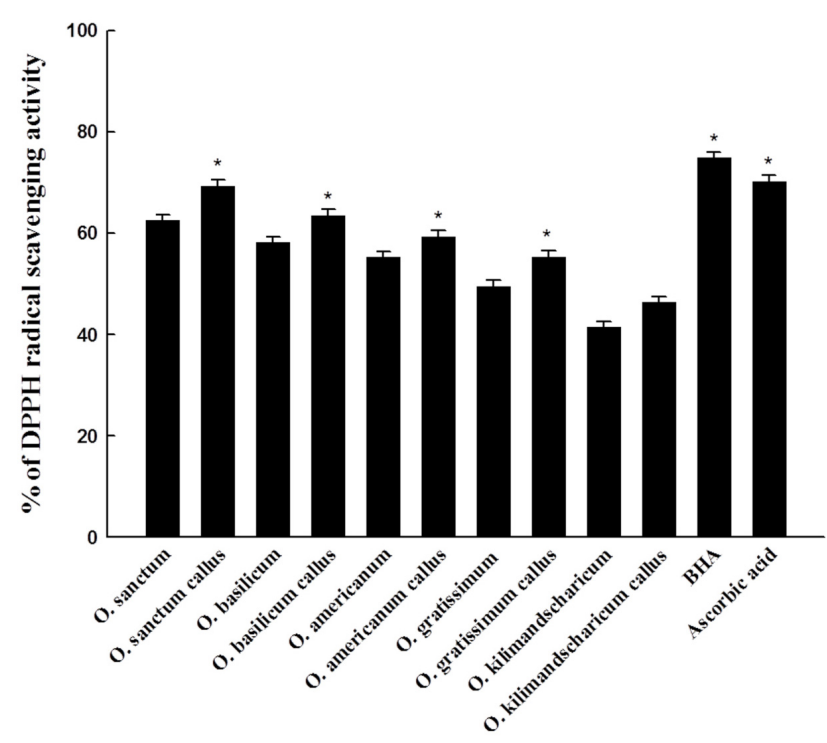

Fig. 2 DPPH radical scavenging activities of five different Ocimum species leaves and their in vitro leaf callus extracts. Data are presented as mean $\pm \mathrm{SD}$ of each of three replicates $(n=3)$ * indicates a significant $(\mathrm{p}<0.05)$ increase in callus extract compare to their respective in vivo leaf extracts along with positive controls. BHA - Butylated hydroxyl anisole

the antiradical power of the antioxidant activity by measuring the decrease in absorbance of DPPH at $517 \mathrm{~nm}$.

As can be seen in Fig. 2, all the Ocimum species extracts at a dose of $1.0 \mathrm{mg} / \mathrm{ml}$ were capable of scavenging $\mathrm{DPPH}^{\circ}$ free radicals. From the percentage scavenging values, it can be seen that the $O$. sanctum leaf extract was

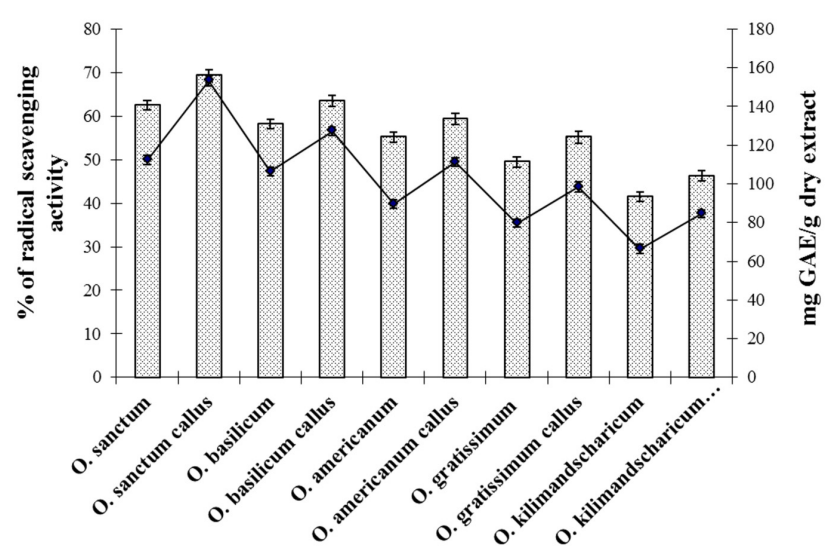

Fig. 3 Relationship between DPPH radical scavenging activity and total phenolic contents of five different Ocimum species leaves and their in vitro leaf callus extracts. Data are presented as mean $\pm \mathrm{SD}$ of each of three replicates $(n=3)$

the most potent scavenger $(62.5 \%)$ followed by $O$. basilicum $(58.2 \%),>O$. americanum $(55.2 \%)>O$. gratissimum $(49.5 \%)$, $>$ O. kilimandscharicum (41.5\%). Further, the callus extracts from all the Ocimum species showed more scavenging activity when compared to the leaf extracts. Ocimum sanctum leaf callus showed highest scavenging activity at $69.3 \%$, followed by Ocimum basilicum 63.5\%, Ocimum americanum (59.3\%), Ocimum gratissimum (55.2\%), and Ocimum kilimandscharicum (46.3\%). None of the Ocimum species extracts were as effective DPPH - scavengers as the positive controls BHA (74.87\%) and ascorbic acid (70.2\%). Further in this study, a good linear correlation $\left(\mathrm{R}^{2}=0.783\right)$ was obtained between the concentration of the total phenolic content and the scavenging activity of the DPPH - radical of each extract (Fig. 3). These results indicate that the radical scavenging capacity of each extract may be related to the concentration of phenolics. The antiradical activity of phenolic compounds depends on their molecular structure, i.e. on the availability of the phenolic hydrogens that can form phenoxyl radicals via hydrogen donation (Ramarathnam et al. 1997). A linear correlation between radical scavenging activity and phenolic concentration has been reported in an extensive range of vegetables, fruits, and beverages (Velioglu et al. 1998).

\section{Effect of extracts on FRAP assay}

Determination of ferric/antioxidant power FRAP is a direct and simple test for measuring the antioxidant capacity, and the reaction is reproducible and linearly related to the molar concentration of the antioxidant present. This method was initially developed to assay the plasma antioxidant capacity but can be used for plant extracts as well. It is based on 


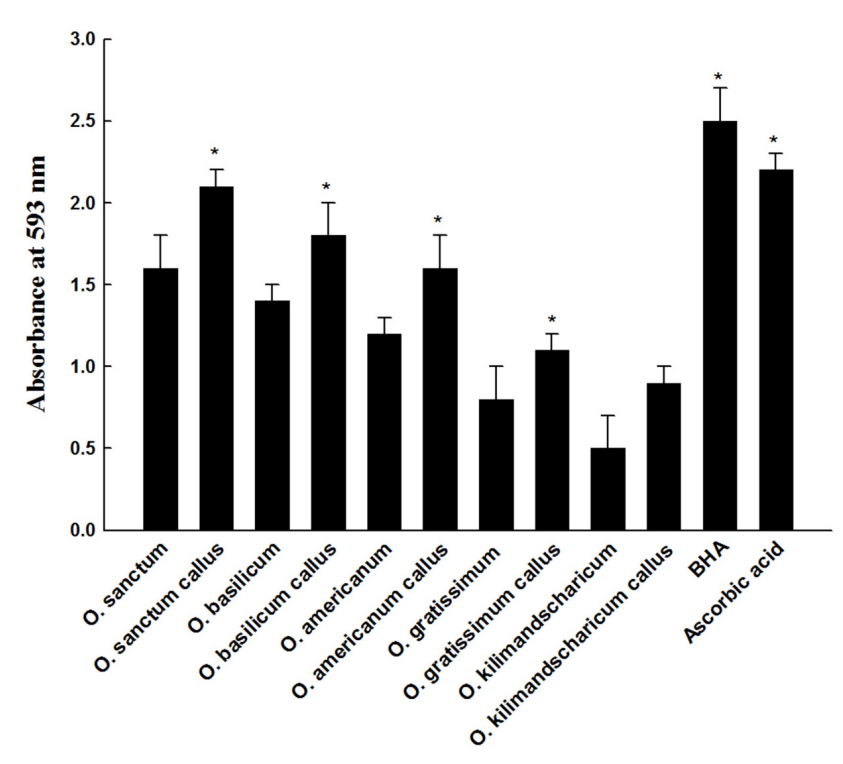

Fig. 4 Reducing power ability of five different Ocimum species leaves and their in vitro leaf callus extracts. Data are presented as mean $\pm \mathrm{SD}$ of each of three replicates $(\mathrm{n}=3)$. $*$ indicates a significant $(\mathrm{p}<0.05)$ increase in callus extract compare to their respective in vivo leaf extracts along with positive controls. BHA - Butylated hydroxyl anisole

comparison of the total amount of antioxidant to the reducing capacity of the sample. The total antioxidant potential of the sample was determined using the ferric reducing ability (FRAP) assay (Benzie and Strain 1996) as a measure of the "antioxidant power". This assay measures the change in absorbance at $593 \mathrm{~nm}$ owing to the formation of a blue colored $\mathrm{Fe}^{2+}$-tri-pyridyltriazine compound from the colorless oxidized $\mathrm{Fe}^{3+}$ form by electron donating antioxidants.

A hierarchy in the reducing capacity of samples could be observed in the order of $O$. sanctum $>O$. basilicum $>$ $O$. americanum $>O$. gratissimum $>O$. kilimandscharicum (Fig. 4). Interestingly, all callus extracts showed significantly increased activities compared to their respective in vivo leaves. This could be due to a higher accumulation of phenolics in the callus extracts.

\section{Effect of Extracts on $\mathrm{Fe}^{2+}$ Chelating Activity}

The ability to chelate transition metals can be considered an imperative antioxidant mode of action. The ferrous state of iron accelerates lipid oxidation by breaking down the hydrogen and lipid peroxidases to reactive free radicals via the fenton type reaction. The $\mathrm{Fe}^{3+}$ ion also produces radicals from peroxides, although the rate is tenfold less than that of the $\mathrm{Fe}^{2+}$ ion (Miller and Denis 1998). The $\mathrm{Fe}^{2+}$ ion is the most powerful pro-oxidant among various

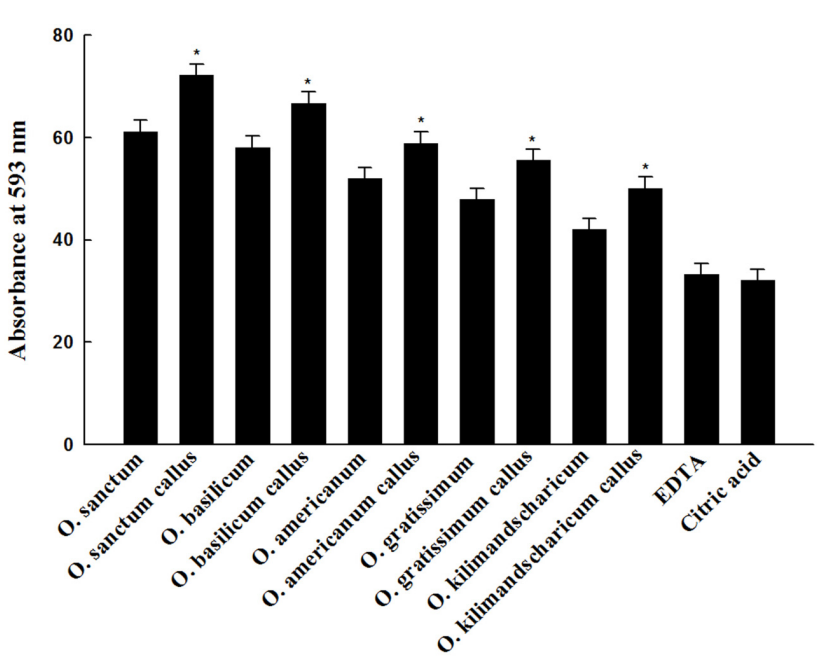

Fig. $5 \mathrm{Fe}^{2+}$ chelating activities of five different Ocimum species leaves and their in vitro leaf callus extracts. Data are presented as mean $\pm \mathrm{SD}$ of each of three replicates $(n=3)$. * indicates a significant $(\mathrm{p}<0.05)$ increase in callus extract compare to their respective in vivo leaf extracts along with positive controls. EDTA - Ethylenediamine tetra acetic acid

species of metal ions (Halliwell and Gutteridge 1984). Ferrozine is a chelating reagent used to indicate the presence of a chelator in the reactionsystem. Ferrozine forms a complex with free $\mathrm{Fe}^{2+}$ ions but not with $\mathrm{Fe}^{2+}$ bound to electron donating molecules. In the presence of chelating agents, the complex formation of ferrous and ferrozine is disrupted, resulting in a decrease in the red color of the complex. Measuring the color reduction, therefore, allows the estimation of the metal chelating activity of the coexisting chelator (Yamaguchi et al. 2000).

Therefore, this study was conducted to determine the ability of five different Ocimum leaf extracts and in vitro callus (induced from each explants) extracts to chelate iron (II) ions. The chelating activity of all the extracts of $1 \mathrm{~m}$ $\mathrm{g} \cdot \mathrm{ml}$ on $\mathrm{Fe}^{2+}$ was determined. The chelating activity of the methanol extract of all the Ocimum species with the concentration of $1 \mathrm{mg} \cdot \mathrm{ml}$ was higher than that of EDTA at $0.01 \mathrm{mM}$ and citric acid at $0.025 \mathrm{mM}$.

It was reported that the chelating activity of a particular extract is mainly due to the presence of chelated metal ions or suppressed reactivity, by occupying all coordination sites of metal ion, by the antioxidants present in the extract (Mahoney and Graf 1986). Similarly, in the present study, the increased chelating activity of the callus extract could be due to the abundant presence of compounds with antioxidant actitivity. Therefore, it may be used as an effective agent in delaying the $\mathrm{Fe}^{2+}$ - catalyzed lipid oxidation. 


\section{$\beta$-Carotene-linoleic acid bleaching}

Iron (III) reduction and synthetic free radical scavenging models are valuable tools to indicate the potential antioxidant activity of plant extracts. However, these systems do not use foods or biologically relevant oxidizable substrates, so a direct protective activity of an extract can be determined (Dorman et al. 2003). Therefore, it was important to assess the inhibitory effect of Ocimum species extracts on oxidation of $\beta$-carotene. In this assay, oxidation of linoleic acid produces hydroperoxide-derived free radicals, which attack the chromophore of $\beta$-carotene, resulting in a bleaching of the reaction emulsion. An extract capable of hindering the oxidation of $\beta$-carotene may be described as a free radical (Liyana-Pathirana and Shahidi 2006).

As can be seen in Fig. 6, all the extracts were able to inhibit the bleaching of $\beta$-carotene by scavenging linoleatederived free radicals. The order of efficacy was $O$. sanctum (leaf - 76.4, callus - 81.2\%) $>O$. basilicum (leaf - 68.4, callus - 76.2\%), $>O$. americanum (leaf - 61.2, callus $68.3 \%$ ) $>$ O. gratissimum (leaf -56.3 , callus $-65.2 \%$ ) $>$ O. kilimandscharicum (leaf - 48.2, callus - $55.3 \%$ ). None of the extracts were as effective as the positive control BHA (93.5 $\pm 2.1 \%)$ and ascorbic acid $(84.6 \pm 2.1 \%)$. It has been suggested that the polarity of an extract is important in generating water:oil emulsions, viz., non-polar extracts are more effective antioxidants than polar extracts due to a 'concentrating effect' within the lipid phase (Porter 1986). In contrary, we used in this work methanol for

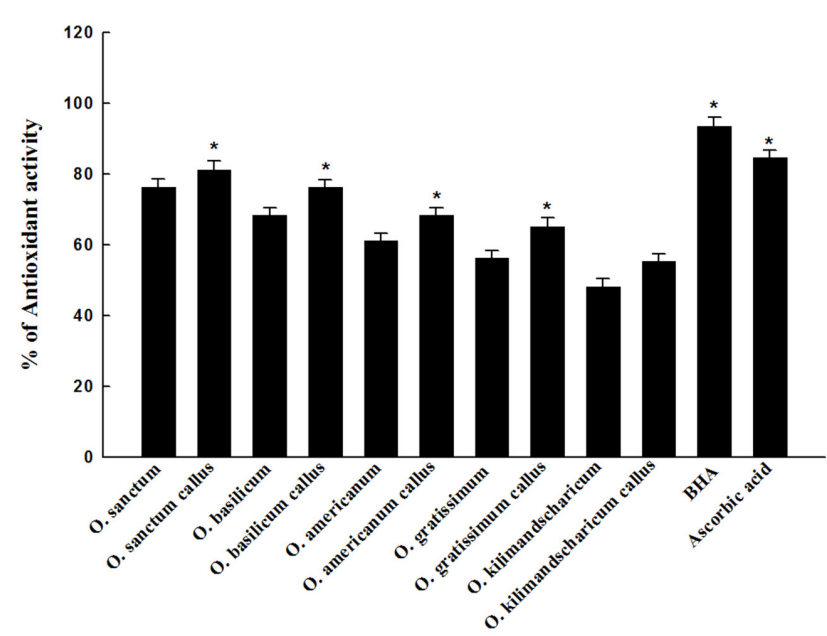

Fig. 6 Antioxidant ability of five different Ocimum species leaves and their in vitro leaf callus extracts by inhibiting the bleaching of $\beta$-carotene. Data are presented as mean \pm SD of each of three replicates $(n=3) . *$ indicates a significant $(\mathrm{p}<0.05)$ increase in callus extract compare to their respective in vivo leaf extracts along with positive controls. BHA - Butylated hydroxyl anisole extraction, which demonstrated appreciable inhibition of $\beta$ -carotene bleaching by scavenging linoleate-derived free radicals. This phenomenon is in agreement with Koleva et al. (2002), who reported inhibition of $\beta$-carotene bleaching by the polar extract of the Sideritis species. According to the $\beta$-carotene-linoleic acid bleaching data, the extracts are capable of scavenging free radicals in a complex heterogenous medium. This suggests that the extracts may have a potential use as antioxidative preservatives in emulsiontype systems.

In conclusion the observed the observed results suggest that callus extracts contains more significant amounts of phenolics, compared to other parts of Ocimum species which tested in this study. In particular, the $O$. sanctum callus extract showed more significant antioxidant ability than other callus extracts, consistent with previous observations. The enhanced antioxidant ability of callus extracts is due to the electron donating ability of the accumulated phenolics. Furthermore, these extracts can be utilized as potential food preservatives against oxidative deterioration, as demonstrated by the $\beta$-Carotene-linoleic acid bleaching assay. Consumption of these extracts may lead to benefits in human health, namely protection against excessive oxidative stress.

\section{Acknowledgment}

This work was carried out with the support of the Cooperative Research Program for Agriculture Science \& Technology Development (Project No. PJ008460022012), Rural Development Administration, Republic of Korea.

\section{References}

Benzie IFF, JJ Strain (1996) The Ferric Reducing Ability of Plasma (FRAP) as a Measure of "antioxidant Power": The FRAP Assay Analytical Biochemistry 239:70-76

Chatterjee A, NC Sukul, S Laskar, S Ghoshmajumdar (1982) Nematicidal principles from two species of lamiaceae. J Nematol 14:118-20

Deshpande RS, HP Tipins (1977) Insecticidal activity of Ocimum basilicum Linn. Pesticides 11:11-12

Dinis TCP, VMC Madeira, LM Almeida (1994) Action of Phenolic Derivatives (Acetaminophen, Salicylate, and 5Aminosalicylate) as Inhibitors of Membrane Lipid Peroxidation and as Peroxyl Radical Scavengers. Archives of Biochemistry and Biophysics 315:161-169

Dorman HJD, A Peltoketo, R Hiltunen, MJ Tikkanen (2003) Characterisation of the antioxidant properties of de- 
odourised aqueous extracts from selected Lamiaceae herbs Food Chemistry 83:255-262

Gyamfi MA, M Yonamine, Y Aniya (1999) Free-radical scavenging action of medicinal herbs from Ghana: Thonningia sanguinea on experimentally-induced liver injuries. General Pharmacology: The Vascular System 32:661-667

Hakkim FL, G Arivazhagan, R Boopathy (2008) Antioxidant property of selected Ocimum species and their secondary metabolite content. Journal of Medicinal Plants Research 2:250-257

Hakkim FL, CG Shankar, S Girija (2007) Chemical Composition and Antioxidant Property of Holy Basil (Ocimum sanctum L.) Leaves, Stems, and Inflorescence and Their in Vitro Callus Cultures. Journal of Agricultural and Food Chemistry 55: 9109-9117

Halliwell B, JM Gutteridge (1984) Oxygen toxicity, oxygen radicals, transition metals and disease. Biochem J 219 1-14

Hirasa K, M Takemasa (1998) Spice Science Technology. Dekker M. Tokyo Japan

Kaur H, J Perkins (1991) The free radical chemistry of food additives. Aruoma OI and B Halliwell. London / New York, Taylor and Francis Free Radicals and Food Additives pp 17-35

Koleva II, TA van Beek, JPH Linssen, Ad Groot, LN Evstatieva (2002) Screening of Plant Extracts for Antioxidant Activity: a Comparative Study on Three Testing Methods. Phytochemical Analysis 13:8-17

Liyana-Pathirana CM, F Shahidi (2006) Antioxidant properties of commercial soft and hard winter wheats (Triticum aestivum L.) and their milling fractions. Journal of the Science of Food and Agriculture 86:477-485

Lutomski J. (2002) Znaczenie ziolw terapii i dietetyce. Herba polonica 48:300-310

Mahoney JR, E Graf (1986) Role of Alpha-Tocopherol, Ascorbic Acid, Citric Acidand EDTA as Oxidants in Model Systems. Journal of Food Science 51:1293-1296

Miller, Denis (1998) Food Chemistry: A Laboratory Manual. Wiley, Wiley (New York): ix pp 153

Murashige T, F Skoog (1962) A Revised Medium for Rapid Growth and Bio Assays with Tobacco Tissue Cultures. Physiologia Plantarum 15:473-497

Ozkan G, O Sagdic, M Ozcan (2003). Inhibition of Pathogenic
Bacteria by Essential Oils at Different Concentrations. Food Science and Technology International 9(2):85-88

Paula C, G Santos, M Rosa, PB Seabra, M Andrade, F Fernades (2002) Phenolicantioxidant compounds produced by in vitro shoots of sage (Salvia officinalis L.). Plant Sci 162:981

Porter WL(1986) Recent trends in food applications of antioxidants. Simic MG and M Karel New York, Plenum Press Autoxidation in Food and Biological Systems pp 295-365

Ramarathnam N, H Ochi, M Takeuchi (1997) Antioxidant defense system in vegetable extracts. Shahidi F. Japan Natural Antioxidants; Chemistry, Health Effects and Application pp 76

Simon JE, MR Morales, WB Phippen, RF Vieira, Z Hao (1999) Basil: A Source of Aroma Compounds and a Popular Culinary and Ornamental Herb. Perspectives on new crops and new uses

Singleton VL, R Orthofer, RM Lamuela-Raventcjs, P Lester (1999) Analysis of total phenols and other oxidation substrates and antioxidants by means of folin-ciocalteu reagent. Methods in Enzymology Academic Press Volume 299:152-178

Szabo E, A Thelen, M Petersen. (1999). Fungal elicitor preparations and methyl jasmonate enhance rosmarinic acid accumulation in suspension cultures of Coleus blumei. Plant Cell Rep 18: 485

Szeto YT, B Tomlinson, IF Benzie (2002) Total antioxidant and ascorbic acid content of fresh fruits and vegetables: implications for dietary planning and food preservation. Br J Nutr 87:55-9

Vadivel V, H Biesalski (2011) Effect of certain indigenous processing methods on the bioactive compounds of ten different wild type legume grains. Journal of Food Science and Technology pp 1-12

Velioglu YS, G Mazza, L Gao, BD Oomath (1998) Antioxidant activity and total phenolics in selected fruits, vegetables, and grain products. J Agric Food Chem 46:4113

Verpoorte R, AW Alferman (2000) Metabolic Engineering of Plant Secondary Metabolism. Alferman AW Dordrecht Netherlands Kluwer Academic press

Yamaguchi F, T Ariga, Y Yoshimura, H Nakazawa. (2000). Antioxidative and Anti-Glycation Activity of Garcinol from Garcinia indica Fruit Rind. Journal of Agricultural and Food Chemistry 48:180-185 